\title{
The cosmic radio dipole and local structure effects
}

\author{
Matthias Rubart ${ }^{1}$, David Bacon ${ }^{2}$ and Dominik J. Schwarz ${ }^{1}$ \\ ${ }^{1}$ Fakultät für Physik, Universität Bielefeld, Postfach 100131, 33501 Bielefeld, Germany \\ email: matthiasr at physik dot uni-bielefeld dot de \\ ${ }^{2}$ Institute of Cosmology and Gravitation, University of Portsmouth, Burnaby Road, \\ Portsmouth PO1 3FX, United Kingdom
}

\begin{abstract}
We investigate the contribution of a local over- or under-density to linear estimates of the cosmic dipole. We focus on radio continuum surveys. Recently it was shown that the radio dipole amplitude is larger than expected from the corresponding dipole of the CMB. We show that a significant contribution to this excess could come from local structure.
\end{abstract}

Keywords. Cosmology: observations, large scale structure of the universe, radio surveys, peculiar motion

\section{Dipole in number counts}

The measured $\mathrm{CMB}$ dipole is $\Delta T=3.36 \pm 0.01 \mathrm{mK}$ in the direction $(\mathrm{RA}, \mathrm{Dec})=$ $\left(168^{\circ},-7^{\circ}\right)$ and thus the velocity of the Solar system has been inferred to be $v=369 \pm$ $1 \mathrm{~km} \mathrm{~s}^{-1}$ (Hinshaw et al. (2009)).

The amplitude of the kinetic radio dipole is given by (Ellis \& Baldwin (1984))

$$
d=[2+x(1+\alpha)](v / c),
$$

where $\alpha$ is the mean spectral index of radio sources and $x$ is the power law index of the corresponding number counts. This dipole is due to spherical aberration as well as the Doppler effect. Using the inferred CMB dipole velocity we expect $d=(5 \pm 1) \times 10^{-3}$, pointing towards the CMB dipole.

Blake \& Wall (2002), Singal (2011), Gibelyou \& Huterer (2012) and Rubart \& Schwarz (2013) (2D and 3D) attempted to determine the dipole in the NRAO VLA Sky Survey (NVSS), as shown in the table.

The estimator used by Blake \& Wall is quadratic, while the other estimators are different implementations of linear estimators, essentially:

$$
\vec{D}=\sum_{i}^{N} \hat{r}_{i}
$$

\begin{tabular}{llllll}
\hline Source & $\begin{array}{l}\text { Flux }> \\
(\mathrm{mJy})\end{array}$ & $\mathrm{N}$ & $\begin{array}{l}\mathrm{RA} \\
\left(^{\circ}\right)\end{array}$ & $\begin{array}{l}\text { Dec } \\
\left(^{\circ}\right)\end{array}$ & $\begin{array}{l}d \\
\left(10^{-3}\right)\end{array}$ \\
\hline $\mathrm{B} \& \mathrm{~W}$ & 25 & 197998 & $158 \pm 30$ & $-4 \pm 34$ & $11 \pm 3$ \\
$\vec{D}_{S}$ & 25 & 184237 & $159 \pm 10$ & $-7 \pm 9$ & $22 \pm 6$ \\
$\vec{D}_{G H}$ & 15 & 211487 & $117 \pm 20$ & $+6 \pm 14$ & $27 \pm 5$ \\
$\vec{D}_{2 D}$ & 25 & 195245 & $155 \pm 14$ & & $19 \pm 5$ \\
$\vec{D}_{3 D}$ & 25 & 185649 & $158 \pm 19$ & $-2 \pm 19$ & $18 \pm 6$ \\
\hline expected & from CMB: & 168 & -7 & $5 \pm 1$ \\
\hline
\end{tabular}



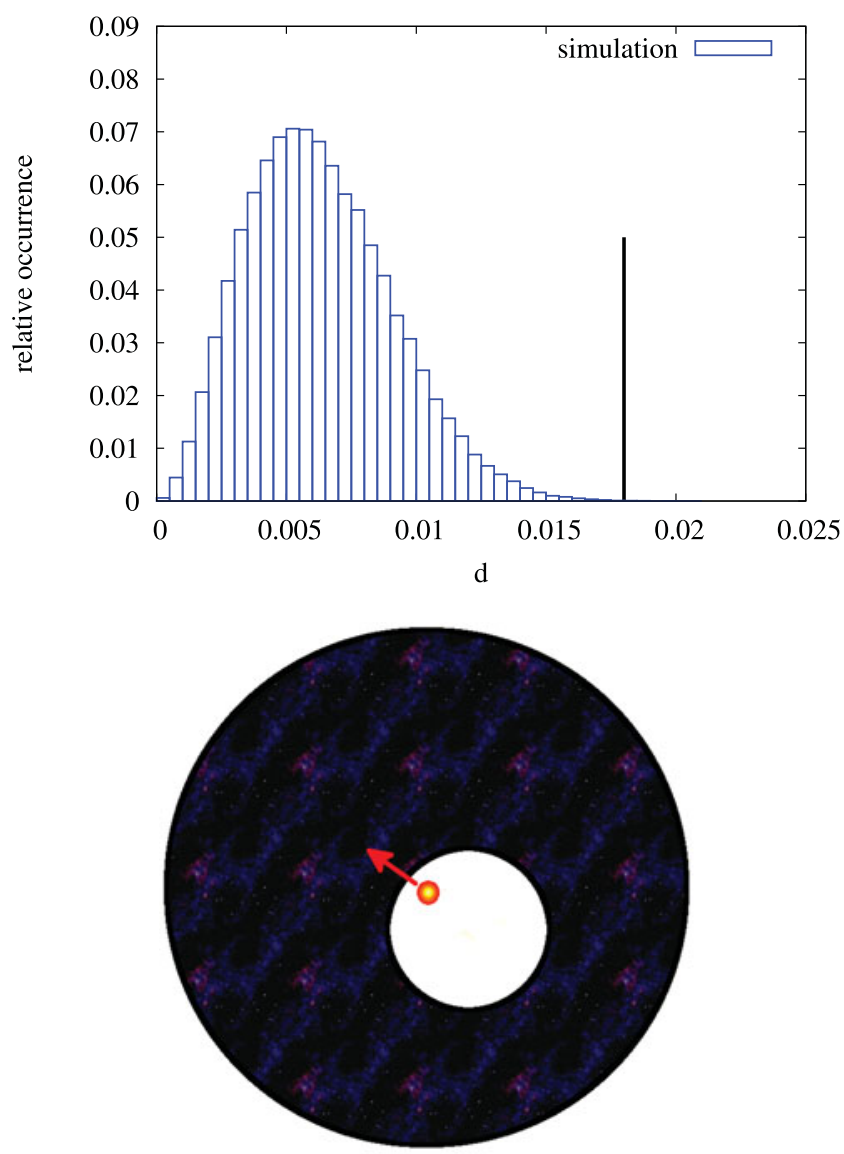

\begin{tabular}{ccc}
\hline $\begin{array}{c}\text { radius of void } \\
\text { (redshift) }\end{array}$ & $\begin{array}{c}\text { relative density } \\
\text { (in percent) }\end{array}$ & $\begin{array}{c}\text { dipole } \\
\left(10^{-3}\right)\end{array}$ \\
\hline $0.07 R_{H}$ & 66 & $2.1 \pm 0.1$ \\
$0.15 R_{H}$ & 66 & $6.9 \pm 0.1$ \\
$0.11 R_{H}$ & 40 & $7.2 \pm 0.1$ \\
\hline
\end{tabular}

The general direction of the radio dipole coincides with the expectation from the CMB. The amplitudes, especially for the linear estimators, are well above the expectation. In order to investigate the significance of this excess, Rubart \& Schwarz (2013) simulated the expected radio sky:

Our simulations show that the observed excess of the amplitude $d$ cannot be explained by shot noise or bias alone.

\section{Local structure dipole}

There have been studies claiming that the local Universe has an untypically low density of galaxies on $300 \mathrm{Mpc}$ scales, e.g. Keenan et al. (2013). We expect to see more galaxies in one direction than in the other, assuming we live in such region. It is likely that the Local Group moves towards this direction, due to a gravitational pull.

The results of Rubart et al. (2014) shows a structural dipole component, which affects 


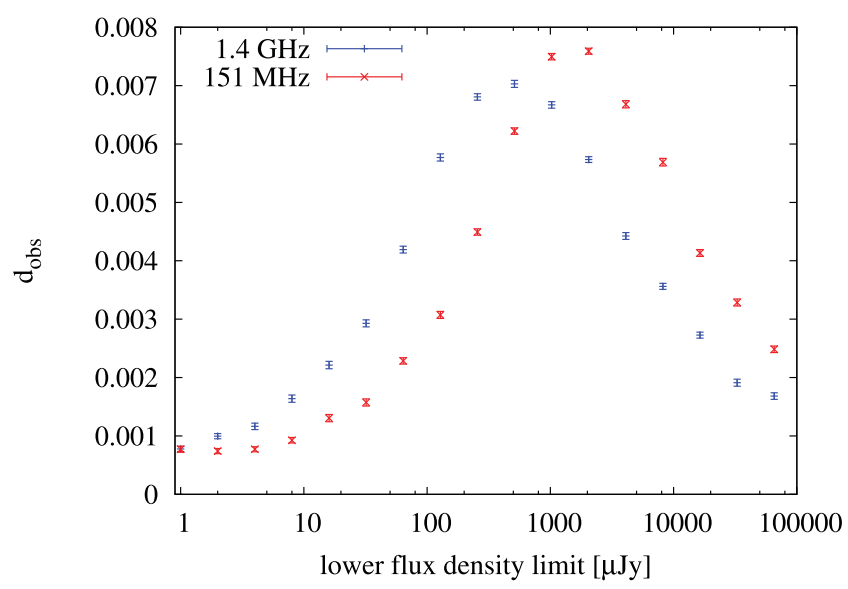

the measured radio dipole. This effect depends on the size of the void and its density contrast.

While the CMB dipole is caused by the motion of the Sun relative to the CMB, the radio dipole can be expected to also receive contributions from the galaxy distribution (structure dipole). Therefore we expect the structural contribution to add up with the velocity dipole resulting in a larger dipole amplitude in radio surveys.

It turns out that there is also a dependence on the lower flux density limit and on the survey frequency band (shown in the graph above for a radius of $0.07 R_{h}$ and $\delta=-0.33$ density contrast). This dependence can be used to distinguish the kinetic dipole from the structural one.

\section{Discussion}

We find that simple void models have a significant effect on the cosmic radio dipole estimation. The dipole amplitude measured by the linear estimator of a void of radius $0.07 R_{\mathrm{H}}$ is expected to be $d_{\mathrm{void}}=(2.1 \pm 0.1) \times 10^{-3}$. The discrepancy between radio and CMB dipole measurements can be relaxed by such a contribution, but the difference cannot be explained completely. In forthcoming surveys (Lofar MSSS \& Tier 1, EMU and SKA surveys), the effect of structures will become more important, due to lower flux limits.

\section{References}

Blake, C. \& Wall, J. 2002, Nature, 416, 150

Ellis, G. F. R. \& Baldwin J. E. 1984, MNRAS, 206, 377

Gibelyou, C. \& Huterer, D. 2012, MNRAS, 427, 1994

Hinshaw, G., Weiland, J. L., Hill, R. S., et al. 2009, ApJS, 180, 225

Keenan, R. C., Barger, A. J., \& Cowie, L. L. 2013, ApJ, 775, 62

Rubart, M. \& Schwarz, D. J. 2013, A\&A, 555, A117

Rubart, M., Schwarz, D. J., \& Bacon, D. 2014, A\&A, 565, A111

Singal, A. K. 2011, ApJ, 742, L23 